\title{
Development and characterization of fourteen novel microsatellite markers for the chestnut short-tailed fruit bat (Carollia castanea), and cross-amplification to related species
}

\author{
Katherine A Cleary ${ }^{\text {Corresp., }}{ }^{1}$, Lisette P Waits ${ }^{1}{ }^{\text {, Paul A Hohenlohe }}{ }^{2}$ \\ 1 Department of Fish and Wildlife Sciences, University of Idaho, Moscow, ID, United States \\ 2 Department of Biological Sciences, Institute of Bioinformatics and Evolutionary Studies, University of Idaho, Moscow, ID, United States \\ Corresponding Author: Katherine A Cleary \\ Email address: katecleary98@gmail.com
}

Rapid anthropogenic land use change threatens the primary habitat of the Chestnut shorttailed bat (Carollia castanea) throughout much of its range. Information on population genetic structure can inform management strategies for this widespread frugivorous bat, and effective protection of $C$. castanea will also benefit the more than 20 mutualistic plant species of which this bat is the primary seed disperser. To facilitate understanding of population genetic structure in this species, fourteen novel microsatellite markers were developed using restriction-site-associated DNA libraries and Illumina sequencing and tested on 28 individuals from 13 locations in Costa Rica. These are the first microsatellite markers developed for $C$. castanea. All loci were polymorphic, with number of alleles ranging from 2-11 and average observed heterozygosity of 0.631 . Markers were also cross-amplified in three additional frugivorous bat species threatened by habitat loss and fragmentation: Sowell's short-tailed bat (Carollia sowelli), Seba's short-tailed bat (Carollia perspicillata), and the Jamaican fruit bat (Artibeus jamaicensis), and 10, 11, and 8 were polymorphic, respectively. 
1 Development and characterization of fourteen novel microsatellite markers for the chestnut

2 short-tailed fruit bat (Carollia castanea), and cross-amplification to related species

3 Cleary, Katherine ${ }^{1 *}$, Waits, Lisette ${ }^{1}$, Hohenlohe, Paul ${ }^{2}$

41 Department of Fish and Wildlife Sciences, University of Idaho, Moscow, ID

52 Department of Biological Sciences, Institute of Bioinformatics and Evolutionary Studies, University of Idaho,

6 Moscow, ID

$7 \quad$ *Corresponding author, Department of Fish and Wildlife Sciences, University of Idaho, 875 Perimeter Drive MS

8 1136, Moscow ID 83844-1136, email: katecleary98@gmail.com, fax: 208-885-5534 


\section{ABSTRACT}

Rapid anthropogenic land use change threatens the primary habitat of the Chestnut shorttailed bat (Carollia castanea) throughout much of its range. Information on population genetic structure can inform management strategies for this widespread frugivorous bat, and effective protection of $C$. castanea will also benefit the more than 20 mutualistic plant species of which this bat is the primary seed disperser. To facilitate understanding of population genetic structure in this species, fourteen novel microsatellite markers were developed using restriction-site-associated DNA libraries and Illumina sequencing and tested on 28 individuals from 13 locations in Costa Rica. These are the first microsatellite markers developed for $C$. castanea. All loci were polymorphic, with number of alleles ranging from 2-11 and average observed heterozygosity of 0.631. Markers were also cross-amplified in three additional frugivorous bat species threatened by habitat loss and fragmentation: Sowell's short-tailed bat (Carollia sowelli), Seba's short-tailed bat (Carollia perspicillata), and the Jamaican fruit bat (Artibeus jamaicensis), and 10,11, and 8 were polymorphic, respectively.

\section{INTRODUCTION}

The Chestnut short-tailed bat (Carollia castanea) is a frugivorous bat which inhabits tropical forests from Honduras to Bolivia, and is the primary seed disperser of many pioneer plant species found in regenerating forests (Lopez and Vaughan 2007). Throughout this species' range, rapid conversion of native forest cover to agriculture is driving habitat loss and fragmentation, which threatens the ability of $C$. castanea populations to maintain genetic connectivity. C. castanea has a small body size, limited home range of $<7$ ha, and low wing loading, all of which are associated with lower vagility and increased vulnerability to fragmentation in bats (Bonaccorso et al. 2006, Meyer et al. 2008, Meyer et al. 2009). As a result, populations of $C$. castanea in fragmented agricultural landscapes are at risk of interrupted gene flow, genetic drift, and inbreeding, which reduce genetic diversity and adaptive capacity in the face of future perturbations (Willi et al. 2007, Mendez et al. 2011). 
To date, only one study has used molecular markers to evaluate the impact of ongoing land use change on C. castanea. Ripperger et al (2014) sequenced the mitochondrial d-loop of 173 C. castanea individuals sampled from 10 plots in continuous forest and remnant forest patches in a fragmented agricultural landscape in northern Costa Rica, and found no evidence of significant genetic structure. However, mitochondrial markers have a much slower mutation rate than nuclear DNA markers, and are thus less capable of detecting recent response to landscape change (Wang et al 2010). Since forest fragmentation and agricultural expansion in tropical regions have happened very recently on an evolutionary timescale, we would expect that only neutral nuclear DNA markers with high rates of mutation such as microsatellites would already show a response to these processes. Despite the utility of microsatellite markers for evaluating population responses to recent land use change, no microsatellite loci have previously been developed for $C$. castanea. In fact, of the seven currently recognized species in the Carollia genus (Velazco 2013), microsatellite markers have only been developed for one, Carollia brevicauda (Bardeleben et al 2007); these authors also successfully cross-amplified 8 microsatellites to $C$. castanea.

The goal of this study was to develop the first microsatellite markers specifically for $C$. castanea using Illumina high-throughput sequencing, to fully characterize these markers using a small sample of individuals from Costa Rica, and to evaluate the transferability of these microsatellites to three other co-distributed bat species: Sowell's short-tailed bat (Carollia sowelli), Seba's short-tailed bat (Carollia perspicillata), and the Jamaican fruit bat (Artibeus jamaicensis). These novel markers can be used to quantify population genetic structure, identify populations that have become genetically isolated due to habitat loss and fragmentation, and evaluate correlations between genetic diversity, gene flow, and land use in fragmented agricultural landscapes. Future studies can also use these markers to increase understanding of mating and dispersal strategies in C. castanea; preliminary evidence of female-biased dispersal has been found in this species using mitochondrial DNA markers (Ripperger et al 2014), which is very rare in mammals (Greenwood 1980). 


\section{MATERIALS AND METHODS}

Samples of $C$. castanea were collected in 13 remnant forest patches in the San Juan-La Selva biological corridor in northern Costa Rica (Table 1), where conversion of tropical lowland forest to agriculture has led to widespread habitat loss and fragmentation. Bats were captured using mist nets, and tissue samples of the uropatagium were collected using a $2 \mathrm{~mm}$ diameter circular biopsy tool and stored in a $2 \mathrm{~mL}$ tube with lysis buffer (50 mM Tris pH 8.0, $50 \mathrm{mM}$ EDTA, $50 \mathrm{mM}$ sucrose, $100 \mathrm{mM} \mathrm{NaCl}, 1 \%$ SDS) (Faure 2009). Our capture and handling procedures were approved by the University of Idaho's Animal Care and Use Committee (protocol \#201131), and all field work was conducted with permission from the Costa Rican Ministry of Energy and the Environment (permit number: R-005-2013-OT-CONAGEBIO).

Genomic DNA was extracted from tissue samples of three individuals using the Qiagen Blood and Tissue Kit. Libraries were prepared using a restriction-site-associated DNA approach (Etter et al 2011). In brief, genomic DNA was digested with a restriction enzyme, and an adapter containing a $6 \mathrm{bp}$ long RAD tag and both forward amplification and Illumina sequencing priming sites was ligated to the fragments. All fragments were pooled, sheared, and size selected. A second adapter was then ligated to the size selected fragments; this adapter is designed to ensure that only P1 adapter-ligated RAD tags will be amplified during the final amplification step. Final prepared libraries were run on one lane of an Illumina ${ }^{\circledR}$ MiSeq250, which generated 3,179,284 250-bp sequences. Sequences were archived in the NCBI Sequence Read Archive (accession \# SRP082144). Data was de-multiplexed and quality-cleaned using Stacks V.1.21 (Catchen et al 2013). A total of 2,300,295 cleaned sequences were run through the program QDD V.3.1 (Meglecz et al 2014) to identify microsatellites, filter out redundant sequences, and design primers. This process identified 10, 558 sequences containing at least one microsatellite.

All sequences were screened using strict criteria to select only perfect microsatellites with di- or tetranucleotide motifs, at least five repeats, and low alignment scores with known transposable elements. From the 656 candidate loci identified with these criteria we selected 32 high-quality loci to test for amplification. Unlabeled forward and reverse primers for these loci were synthesized through Applied Biosystems. Primers were diluted to a 10uM solution containing both forward and reverse primers, and tested on eight $C$. castanea individuals from 
91 eight different remnant forest patches. Individual amplifications were performed in a $7 \mu \mathrm{L}$ reaction containing $2 \mu \mathrm{L}$ template DNA (at 7ng/ $\mu \mathrm{L}$ ), 2X Qiagen Multiplex PCR Master Mix, 0.5X $\mathrm{Q}$ solution, and $0.10 \mu \mathrm{L}$ of each $10 \mu \mathrm{M}$ primer solution. Cycling conditions consisted of a 15 minute initial denaturation at $95^{\circ} \mathrm{C}$, followed by 15 cycles of a touchdown protocol of $94^{\circ} \mathrm{C}$ for $30 \mathrm{~s} ; 63^{\circ} \mathrm{C}$ for $90 \mathrm{~s} ; 72^{\circ} \mathrm{C}$ for $60 \mathrm{~s}$, and then 20 additional cycles of $94^{\circ} \mathrm{C}$ for $30 \mathrm{~s} ; 57^{\circ} \mathrm{C}$ for $90 \mathrm{~s} ; 72^{\circ} \mathrm{C}$ for 60s.

Amplification products were examined for polymorphism using standard gel electrophoresis with $3 \%$ agarose gels. Twenty loci were identified as potentially polymorphic. For these twenty loci, fluorescent labeled forward primers and unlabeled reverse primers were synthesized through Integrated DNA technologies and Applied Biosystems. Using the same amplification reactions and cycling conditions as for the previous step, these loci were amplified and amplification products were separated on an Applied Biosystems 3130xI Analyzer with LIZ500 internal size standard, and scored using GeneMapper 5 (Applied Biosystems). Fourteen of the twenty loci were identified as definitively polymorphic. These loci were multiplexed into two reactions (Table 2) and tested on 20 new C. castanea individuals. To obtain estimates of population genetic parameters representative of the study area, we selected these individuals from thirteen different patches across the study area: the same eight remnant forest patches from the previous step, plus an additional five patches (Table 1). Cycling conditions were the same as used above for both multiplexes. Amplification products were separated on an Applied Biosystems 3130xI Analyzer with LIZ500 internal size standard, and scored using GeneMapper 5 (Applied Biosystems). To validate scoring methods, the distribution of raw allele sizes was visualized and the best bin sets for each locus were generated using Autobin v.09 (Figure S1). All loci were tested twice using DNA from the same $C$. castanea samples to ensure reliable results.

5 Number of alleles per locus $\left(\mathrm{N}_{\mathrm{A}}\right)$, observed heterozygosity $\left(\mathrm{H}_{\mathrm{O}}\right)$, expected heterozygosity $\left(H_{E}\right)$, and tests for departures from Hardy-Weinberg equilibrium $\left(P_{H W E}\right)$ were calculated in GenAIEx 6.5 (Peakall and Smouse 2012), and loci were screened for null alleles in CERVUS 3.0.7 (Kalinowski 2007). To assess baseline frequencies of each allele across the study area, GenAlEx 6.5 was used to calculate allele frequencies across all samples. Next, program STRUCTURE v 
120 2.3.4 (Pritchard et al 2000) was used to test for genetic structure in the data. We chose an

121 admixture model with correlated allele frequencies; this model is appropriate for our system

122 because since land use change in the study area is a recent event we expected that allele

123 frequencies in the remnant forest patches would still be fairly similar (Falush et al 2003). Since

124 the samples were collected from 13 different forest patches and it is possible that $C$. castanea

125 populations in these patches represent 13 distinct genetic groups, we tested all values of $\mathrm{K}$

126 between one and 13. As recommended by Gilbert et al (2012), we ran the model for 100,000

127 generations, with a 100,000 generation burn-in, and confirmed this number of generations was

128 adequate by checking for convergence of alpha, F, D, and log likelihood. We ran three

129 independent replicate runs using the same model settings.

130 All fourteen loci were also tested for amplification and polymorphism in two individuals

131 of Carollia sowelli, three individuals of Carollia perspicillata, and three individuals of Artibeus

132 jamaicensis. These species were chosen because like $C$. castanea they face threats from habitat

133 destruction throughout their range and together are key seed dispersers for hundreds of

134 species of Neotropical plants (Ortega and Castro-Arellano 2001, Thies and Kalko 2004, Lopez

135 and Vaughan 2007). Samples from all three species were collected in the same remnant forest

136 patches where the $C$. castanea samples were collected (Table 1), and under the same handling

137 and collection permits as described above. In the laboratory, the PCR conditions were the same

138 as used for C. castanea, but loci were tested separately to avoid the potential problem of

139 overlapping alleles caused by shifting size ranges in the new species. Population genetic

140 analyses were not conducted on these data due to the small sample size for each species.

\section{RESULTS AND DISCUSSION}

We successfully developed and characterized 14 novel microsatellite markers for $C$. castanea, of which 13 are likely to be useful for future research on this species. In addition, our tests of cross-amplification to C. sowelli, C. perspicillata, and Artibeus jamaicensis revealed that a large subset of these loci amplify and are polymorphic in these species as well.

Primer sequences, size range of amplification product, and multiplex assignment for each of the fourteen microsatellite loci are presented in Table 2. All loci were in HWE with the 
148 exception of CC-30 ( $p=0.003$ ) (Table 2$)$, and all loci had null allele frequencies of $<1 \%$ except 149 CC-24 (14\%) and CC-30 (53\%). The null allele rate in CC-24 is moderate and this locus was not 150 out of HWE, so we consider it a reliable marker for use in C. castanea. The marker CC-30 was significantly out of HWE and showed a fairly high null allele rate in these analyses, so it is not likely that this marker will prove to be reliable for use in this species. Excluding these two potentially problematic loci, remaining loci had 2-11 alleles per locus, with an average observed heterozygosity of 0.631 ( \pm 0.227 ) (Table 2 ). These levels of polymorphism and heterozygosity are similar to those found by Bardeleben et al (2007): the loci these authors cross-amplified from C. brevicauda to C. castanea showed 2-18 alleles per locus, with an average observed heterozygosity of 0.69 . Calculation of allele frequencies across the study area revealed that loci CC-10 and CC-30 are the only two loci where a single allele has a frequency of greater than 0.7 (Figure 1).

For all three independent replicate runs in STRUCTURE, $K=1$ had the largest log likelihood (closest to zero) of all tested values. This indicated that the most likely number of genetic groups was one $(K=1)$, with a posterior probability the first run of $\ln \operatorname{Pr}(X \mid K)=0.49$. These results are in line with the findings from Ripperger et al (2014), who used mitochondrial DNA markers and found no significant genetic structure among $C$. castanea populations in the same region. However, it is important to note that since the primary purpose of the present study is to develop new microsatellite markers, only a very small number of individuals were sampled $(n=20)$. It is possible that analyzing additional individuals and individuals from more isolated forest patches could reveal significant genetic structure at the scale of the study area.

The novel microsatellite markers we have developed here will facilitate such future studies of population genetic structure in C. castanea and enable tests of whether levels of genetic diversity and gene flow in isolated populations are correlated with land use change and habitat loss and fragmentation. Understanding the impact of these processes on $C$. castanea is especially important since this bat is known to disperse at least 20 species of Neotropical plants (Lopez and Vaughan 2007). If C. castanea is able to maintain gene flow in fragmented landscapes, then these mutualistic plant species will also have a better chance of maintaining reproductive connectivity, genetic diversity, and recolonization capacity. In addition, these 
177 markers can be used to help resolve persistent taxonomic uncertainty within the Carollia genus

178 (Velazco 2013). Previous studies have used mitochondrial DNA markers to identify cryptic

179 species within C. castanea, including C. benkeithi in Ecuador and Panama (Solari and Baker

180 2006), and an unnamed species from samples collected in Panama (Velazco 2013). Although

181 we are confident that all of the samples used in this study are $C$. castanea since neither of these

182 cryptic species overlaps in range with our study area, this taxonomic uncertainty should be

183 considered when using the microsatellite markers presented here.

184 Our analyses were also successful in determining the utility of these microsatellite

185 markers in the three co-distributed frugivorous bat species. Ten loci amplified and were 186 polymorphic for $C$. sowelli; these represent the first microsatellite markers available for this 187 species (Table 3). Eleven loci amplified and were polymorphic for C. perspicillata, and 8 loci

188 amplified for $A$. jamaicensis, but only 6 were polymorphic (Table 3). Rates of polymorphism for 189 these loci in these three species may be higher than reported here, since loci were tested in a 190 small number of individuals of each species ( $n=2-3$ individuals per species). In addition, allele

191 frequencies reported in Table 3 may not be representative of allele frequencies at these loci in 192 the larger populations, since testing loci in only a few individuals can lead to ascertainment 193 bias. Although microsatellite markers have previously been cross-amplified to C. perspicillata 194 and directly developed for $A$. jamaicensis, these additional markers will add resolution and 195 power to future studies of genetic patterns in these species.

\section{ACKNOWLEDGEMENTS}

197 The authors thank the landowners of Sarapiquí for allowing access to their land and Henry Lara 198 Perez for help with species identification and sample collection. We are grateful to Cody

199 Weinch and Tamara Max for invaluable assistance with developing RAD libraries.

REFERENCES

201 Bardeleben C, Campbell P, Lara M, Moore RL. 2007. Isolation of polymorphic tetranucleotide microsatellite markers for the silky short-tailed bat Carollia brevicauda. Molecular Ecology Notes 7: 63-65. DOI 0.1111/j.1471-8286.2006.01525.x 
204 Bonaccorso FJ, Winkelmann JR, Shin D, Agrawal Cl, Aslami N, Bonney C, Hsu A, Jekielek PE, 205 Knox AK, Kopach SJ, Jennings TD, Lasky JR, Menesale SA, Richards JH, Rutland JA, Sessa AK,

206 Zhaurova L, Kunz TH. 2006. Evidence for Exploitative Competition, Comparative Foraging

207 Behavior and Roosting Ecology of Short-Tailed Fruit Bats (Phyllostomidae). Biotropica 39: 249-

208 256. DOI 10.1111/j.1744-7429.2006.00251.x

209 Catchen J, Hohenlohe PA, Bassham S, Amores A, Cresko WA. 2013. Stacks: an analysis tool kit

210 for population genomics. Molecular Ecology 22: 3124-40. DOI 10.1111/mec.12354

211 Etter PD, Bassham S, Hohenlohe PA, Johnson EA, Cresko WA. 2011. SNP discovery and

212 genotyping for evolutionary genetics using RAD sequencing. In: Molecular Methods for

213 Evolutionary Genetics. Eds: Orgogozo V, Rockman MV. Humana Press, New York, pp. 157-178.

214 Falush D, Stephens M, Pritchard JK. 2003. Inference of population structure: Extensions to

215 linked loci and correlated allele frequencies. Genetics 164:1567-1587.

216 Greenwood PJ. 1980. Mating systems, philopatry and dispersal in birds and mammals. Animal

217 Behavior 28: 1140-1162. DOI 10.1016/s0003-3472(80)80103-5

218 Kalinowski ST, Taper ML, Marshall TC. 2007. Revising how the computer program CERVUS

219 accommodates genotyping error increases success in paternity assignment. Molecular

220 Ecology 16: 1099-1106. DOI 10.1111/j.1365-294x.2007.03089.x

221 Lopez JE, Vaughan C. 2007. Food niche overlap among neotropical frugivorous bats in Costa

222 Rica. Revista de Biología Tropical 55: 301-13. http://www.ncbi.nlm.nih.gov/pubmed/ 18457139

223 Meglécz E, Pech N, Gilles A, Dubut V, Hingamp P, Trilles A, Grenier R, Martin JF. 2014. QDD

224 version 3.1: A user friendly computer program for microsatellite selection and primer design

225 revisited: experimental validation of variables determining genotyping success rate. Molecular

226 Ecology Resources 14: 1302-13. DOI 10.1111/1755-0998

227 Méndez J, Tella L, Godoy JA. 2011. Restricted gene flow and genetic drift in recently

228 fragmented populations of an endangered steppe bird. Biological Conservation 144: 2615-22. 
229 Meyer CFJ, Fründ J, Lizano WP, Kalko EKV. 2008. Ecological correlates of vulnerability to

230 fragmentation in Neotropical bats. Journal of Applied Ecology 45: 381-391. DOI 10.1111/j.1365-

$231 \quad 2664.2007 .01389 . x$

232 Meyer, CFJ, Kalko, EKV, Kerth, G. 2009. Small-Scale Fragmentation Effects on Local Genetic

233 Diversity in Two Phyllostomid Bats with Different Dispersal Abilities in Panama. Biotropica 41:

234 95-102. DOI 10.1111/j.1744-7429.2008.00443.x

235 Peakall R, Smouse PE. 2012. GenAlEx 6.5: genetic analysis in Excel. Population genetic software 236 for teaching and research - an update. Bioinformatics 28: 2537-2539.

237 Pritchard JK, Stephens M, Donnelly P. 2000. Inference of population structure using multilocus 238 genotype data. Genetics 155: 945-959.

239 Ripperger SP, Tschapka M, Kalko EKV, Rodríguez-Herrera B, Mayer F. 2014. Resisting habitat

240 fragmentation, High genetic connectivity among populations of the frugivorous bat Carollia 241 castanea in an agricultural landscape. Agriculture, Ecosystems and Environment 185: 9-15.

242 Solari S, Baker RJ. 2006. Mitochondrial DNA sequence, karyotypic, and morphological variation

243 in the Carollia castanea species complex (Chiroptera: Phyllostomidae) with description of a new 244 species. Occasional Papers, Museum of Texas Tech University 254: 1-16.

245 Velazco, PM. 2013. On the phylogenetic position of Carollia manu Pacheco et al., 2004 246 (Chiroptera: Phyllostomidae: Carolliinae) Zootaxa 3718: 267-276.

247 Wang IJ. 2010. Recognizing the temporal distinctions between landscape genetics and 248 phylogeography. Molecular Ecology 19: 2605-2608.

249 Willi Y, Van Buskirk J, Schmid B, Fischer M. 2007. Genetic isolation of fragmented populations 250 is exacerbated by drift and selection. Journal of Evolutionary Biology 20: 534-42. DOI $251 \quad 10.1111 / \mathrm{j} .1420-9101.2006 .01263 . x$ 


\section{Table $\mathbf{1}$ (on next page)}

Number of samples of each species

Table 1. Number of samples of each species collected from the remnant forest patches in the San Juan-La Selva biological corridor in northern Costa Rica. Latitude and longitude of each patch are given in decimal degrees. 
Table 1 Number of samples of each species collected from the remnant forest patches in the San Juan-La Selva biological corridor in northern Costa Rica. Latitude and longitude of each patch are given in decimal degrees.

\begin{tabular}{lccccll}
\hline Patch & $\begin{array}{l}\text { No. samples } \\
\text { C. castanea }\end{array}$ & $\begin{array}{l}\text { No. samples } \\
\text { C. sowelli }\end{array}$ & $\begin{array}{l}\text { No. samples } \\
\text { C. perspicillata }\end{array}$ & $\begin{array}{l}\text { No. samples } \\
\text { A. jamaicensis }\end{array}$ & Lat & Long \\
\hline 1 & 1 & 0 & 1 & 0 & 10.6624 & -84.1625 \\
2 & 1 & 0 & 0 & 0 & 10.4428 & -84.1080 \\
3 & 1 & 0 & 1 & 0 & 10.4342 & -84.1285 \\
4 & 2 & 0 & 0 & 1 & 10.4076 & -84.1516 \\
5 & 1 & 0 & 0 & 0 & 10.4617 & -84.1537 \\
6 & 2 & 0 & 0 & 0 & 10.4543 & -84.3240 \\
7 & 1 & 0 & 0 & 0 & 10.4110 & -84.2458 \\
8 & 2 & 1 & 1 & 0 & 10.4304 & -84.0931 \\
9 & 1 & 0 & 0 & 1 & 10.5466 & -84.1698 \\
10 & 2 & 0 & 0 & 0 & 10.5874 & -84.1600 \\
11 & 1 & 0 & 0 & 0 & 10.5565 & -84.1816 \\
12 & 3 & 0 & 0 & 0 & 10.5348 & -84.1482 \\
13 & 2 & 1 & 0 & 0 & 10.4313 & -84.0712 \\
\hline
\end{tabular}

1 


\section{Table 2 (on next page)}

Microsatellite loci characteristics

Table 2. Microsatellite loci developed and characterized in 28 Chestnut short-tailed fruit bat (C. castanea) samples from Costa Rica. Fluorescent labels attached to forward primers are in brackets. 
Table 2 Microsatellite loci developed and characterized in 28 Chestnut short-tailed fruit bat (C. castanea) samples from Costa Rica. Fluorescent labels attached to forward primers are in brackets.

\begin{tabular}{|c|c|c|c|c|c|c|c|c|c|}
\hline Locus & $\begin{array}{l}\text { GenBank } \\
\text { Accession No. }\end{array}$ & $\begin{array}{l}\text { Repeat } \\
\text { motif }\end{array}$ & Primer 5'-3' & Range (bp) & MP & $N A$ & $H_{O}$ & $H_{E}$ & $P_{\text {HWE }}$ \\
\hline CC-7 & KX060618 & (AC)13 & $\begin{array}{l}\text { [PET]GAGTAACAAATAAGAGGGAACTGGG } \\
\text { GCAACTGCTCACAACCTGTT }\end{array}$ & $292-300$ & 1 & 5 & 0.800 & 0.715 & 0.385 \\
\hline CC-10 & KX060619 & (AATG)7 & $\begin{array}{l}\text { [FAM]TGCAGGGAAGATGAGAATGAACA } \\
\text { CAGGGCCTGGTGCATAGTAG }\end{array}$ & $116-128$ & 1 & 4 & 0.450 & 0.431 & 0.981 \\
\hline CC-12 & KX060620 & (ACATAT) 12 & $\begin{array}{l}\text { [VIC]ACAGACCAAGAACAGAGCTG } \\
\text { ATGATCTCTGAGCGCTCACA }\end{array}$ & $236-420$ & 1 & 11 & 0.929 & 0.870 & 0.389 \\
\hline CC-13 & KX060621 & $(A G) 6$ & $\begin{array}{l}\text { [NED]CCGAGTCGTTTAGGCTGGTT } \\
\text { GCCCAACCCTGTCTTTGTC }\end{array}$ & $181-185$ & 1 & 2 & 0.500 & 0.455 & 0.658 \\
\hline CC-18 & KX060622 & $(\mathrm{AAGG}) 13$ & $\begin{array}{l}\text { [PET]AGCAGGACGTAAGACAGCAG } \\
\text { TTCCATTTCATTGCTGTGGC }\end{array}$ & 234-245 & 1 & 4 & 0.632 & 0.622 & 0.159 \\
\hline CC-19 & KX060623 & (AC)18 & $\begin{array}{l}\text { [PET]CCCTGCACCAAATCAGCAAT } \\
\text { CTGCCAGCAATGCGTGAATG }\end{array}$ & $120-142$ & 1 & 6 & 0.650 & 0.703 & 0.558 \\
\hline CC-20 & KX060624 & (AT)11 & $\begin{array}{l}\text { [VIC]AGGAAGGGAGTCACCATGGT } \\
\text { CCAACCAGGTGTTAGTGCTA }\end{array}$ & $178-226$ & 2 & 8 & 0.550 & 0.700 & 0.257 \\
\hline CC-23 & KX060625 & $(A G) 21$ & $\begin{array}{l}\text { [NED]CCTTCTATCTGTGACGCTGCT } \\
\text { TCACGCAACAAACAGTAAGTGA }\end{array}$ & $226-256$ & 1 & 10 & 0.750 & 0.781 & 0.898 \\
\hline CC-24 & KX060626 & (ACAG)5 & $\begin{array}{l}\text { [NED]GCAGGACAGGGAGCTTGAAA } \\
\text { ATCATAGAAAGTCGCTGTTGCT }\end{array}$ & $136-140$ & 2 & 2 & 0.368 & 0.494 & 0.267 \\
\hline CC- 25 & KX060627 & (AATG)8 & $\begin{array}{l}\text { [NED]GTCTGTTTCTGCCTCTTTGGG } \\
\text { ATGGGTCACCGTGTCTTAGC }\end{array}$ & $129-141$ & 1 & 4 & 0.600 & 0.554 & 0.932 \\
\hline CC-26 & KX060628 & $(A C) 22$ & $\begin{array}{l}\text { [FAM]GAGGTACGCAGCCAGATGTG } \\
\text { ACTGCTTTCTGGTGCTTCTCA }\end{array}$ & $236-256$ & 1 & 11 & 0.900 & 0.866 & 0.813 \\
\hline CC-27 & KX060629 & $(A C) 21$ & $\begin{array}{l}\text { [FAM]GCAGGGAGTGGAGCATCATC } \\
\text { TGTTGCCAGGTTGTCACAGT }\end{array}$ & 193-209 & 1 & 9 & 0.750 & 0.776 & 0.892 \\
\hline CC-29 & KX060630 & $(A C) 12$ & $\begin{array}{l}\text { [VIC]ACCCTTGCTAGTCTGCCAAC } \\
\text { GAAGGCTCGGTCCTGCTC }\end{array}$ & $220-230$ & 1 & 6 & 0.850 & 0.785 & 0.660 \\
\hline CC-30 & KX060631 & $(A G G G) 7$ & $\begin{array}{l}\text { [VIC]AGGCAAACCCACAGACCAAA } \\
\text { CCAGTCTGTTCTCATTCCCGT }\end{array}$ & $119-131$ & 2 & 3 & 0.100 & 0.329 & 0.003 \\
\hline
\end{tabular}




\section{Table 3(on next page)}

Cross amplification in related species

Table 3. Cross-species amplification success, range in base pairs, and number of alleles (NA) for novel C. castanea loci in three related Phyllostomid species. 
Table 3 Cross-species amplification success, range in base pairs, and number of alleles (NA) for novel $C$. castanea loci in three related Phyllostomid species.

\begin{tabular}{llllllllll}
\hline & \multicolumn{3}{l}{ Carollia sowelli } & \multicolumn{4}{c}{ Carollia perspicillata } & \multicolumn{4}{c}{ Artibeus jamaicensis } \\
Locus & Success & Range (bp) & NA & Success & Range (bp) & NA & Success & Range (bp) & NA \\
\hline CC-7 & $2 / 2$ & $286-296$ & 3 & $3 / 3$ & $286-296$ & 4 & $3 / 3$ & $353-381$ & 5 \\
CC-10 & $2 / 2$ & $124-146$ & 3 & $3 / 3$ & $112-146$ & 6 & $3 / 3$ & $112-138$ & 4 \\
CC-12 & 0/2 & -- & -- & $0 / 3$ & -- & -- & $0 / 3$ & -- & -- \\
CC-13 & $2 / 2$ & $153-163$ & 2 & $3 / 3$ & $153-163$ & 2 & $0 / 3$ & -- & - \\
CC-18 & $2 / 2$ & $234-264$ & 4 & $3 / 3$ & $210-292$ & 5 & $1 / 3$ & $276-276$ & 1 \\
CC-19 & $2 / 2$ & $124-132$ & 2 & $2 / 3$ & $122-132$ & 3 & $2 / 3$ & $116-122$ & 3 \\
CC-20 & $2 / 2$ & $193-263$ & 4 & $3 / 3$ & $165-221$ & 6 & $3 / 3$ & $160-162$ & 2 \\
CC-23 & 0/2 & -- & -- & $0 / 3$ & -- & - & $0 / 3$ & -- & - \\
CC-24 & 0/2 & - & -- & $2 / 3$ & $125-133$ & 2 & $3 / 3$ & $124-124$ & 1 \\
CC-25 & $2 / 2$ & $135-145$ & 3 & $3 / 3$ & $135-145$ & 4 & $0 / 3$ & -- & - \\
CC-26 & $2 / 2$ & $215-217$ & 2 & $3 / 3$ & $215-219$ & 3 & $0 / 3$ & -- & -- \\
CC-27 & $2 / 2$ & $185-197$ & 2 & $3 / 3$ & $185-199$ & 3 & $3 / 3$ & $188-192$ & 3 \\
CC-29 & $2 / 2$ & $229-233$ & 2 & $3 / 3$ & $213-239$ & 4 & $3 / 3$ & $189-203$ & 2 \\
CC-30 & O/2 & -- & -- & $0 / 3$ & -- & -- & $0 / 3$ & -- & -- \\
\hline
\end{tabular}

1 
Figure 1 (on next page)

Baseline allele frequencies for all loci

Figure 1. Baseline allele frequencies for all loci, averaged across all 20 sampled individuals of C. castanea. Loci 7-23 are shown in the top panel and loci 24-30 in the bottom panel 

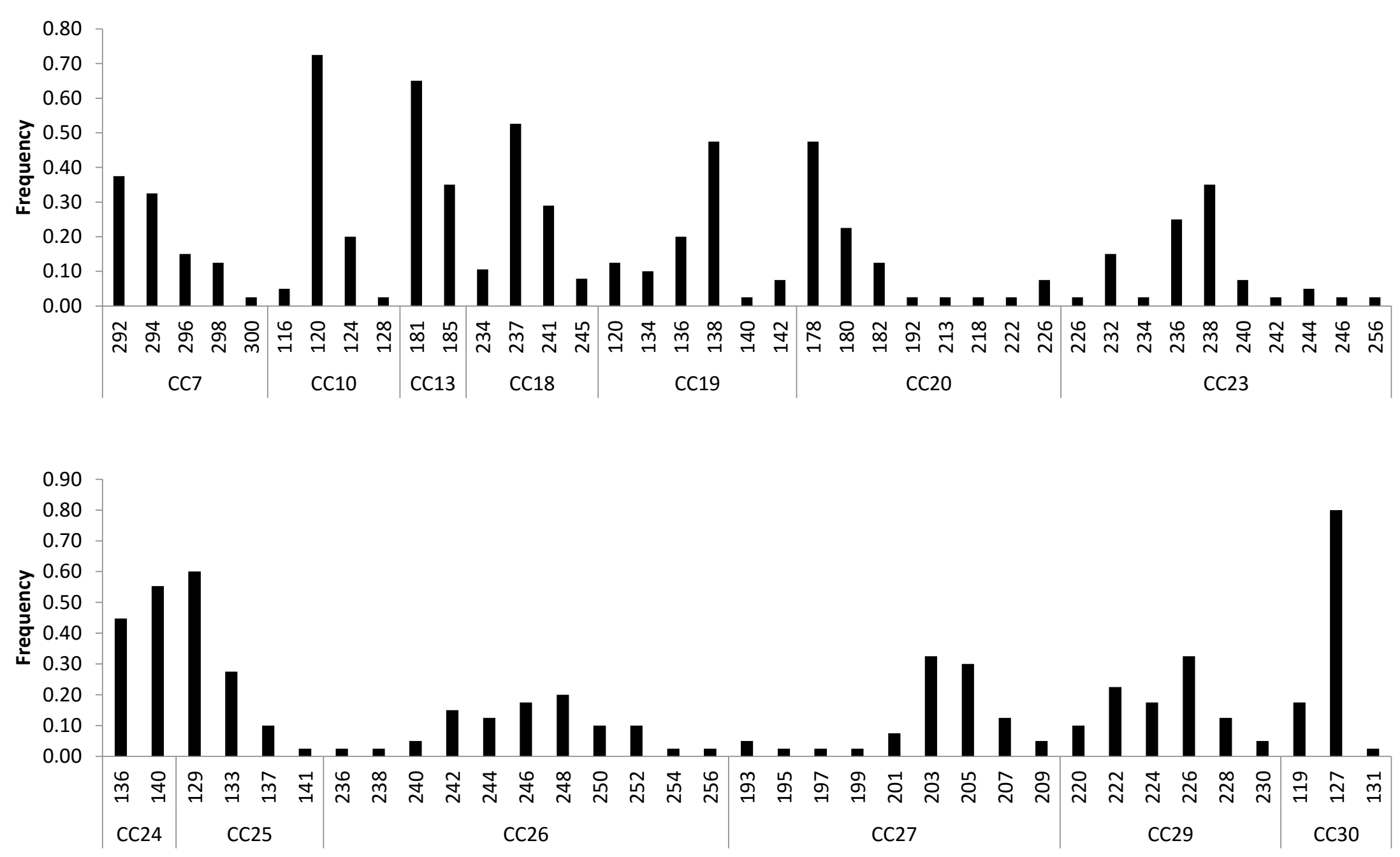